OPEN ACCESS

Edited by:

Olivier Micheau,

Université de Bourgogne, France

Reviewed by:

Jean-Luc Prétet,

Université Bourgogne

Franche-Comté, France

Rosita Accardi-Gheit,

International Agency For Research On

Cancer (IARC), France

Miranda Thomas,

International Centre for Genetic

Engineering and Biotechnology, Italy

Bhudev Chandra Das,

Amity University, India

*Correspondence:

Ahmed Malk

ahmed.malki@qu.edu.qa

Ala-Eddin Al Moustafa

aalmoustafa@qu.edu.qa

Specialty section:

This article was submitted to

Molecular and Cellular Oncology,

a section of the journa

Frontiers in Oncology

Received: 26 February 2020 Accepted: 11 May 2020

Published: 30 June 2020

Citation:

Allouch S, Malki A, Allouch A, Gupta I, Vranic S and Al Moustafa A-E (2020)

High-Risk HPV Oncoproteins and

$P D-1 / P D-L 1$ Interplay in Human

Cervical Cancer: Recent Evidence and

Future Directions.

Front. Oncol. 10:914.

doi: 10.3389/fonc.2020.00914

\section{High-Risk HPV Oncoproteins and PD-1/PD-L1 Interplay in Human Cervical Cancer: Recent Evidence and Future Directions}

\author{
Soumaya Allouch ${ }^{1}$, Ahmed Malki ${ }^{2 *}$, Asma Allouch ${ }^{2}$, Ishita Gupta ${ }^{1,3}$, Semir Vranic ${ }^{1}$ and \\ Ala-Eddin Al Moustafa ${ }^{1,3 *}$ \\ ${ }^{1}$ College of Medicine, QU Health, Qatar University, Doha, Qatar, ${ }^{2}$ Biomedical Science Department, College of Health \\ Sciences, QU-Health, Qatar University, Doha, Qatar, ${ }^{3}$ Biomedical Research Center, Qatar University, Doha, Qatar
}

Cervical cancer is the fourth most common malignancy in women worldwide and a leading cause of cancer-related mortality in developing countries. Important etiological factors in this cancer are high-risk human papillomaviruses (HPV), as roughly 96\% of cervical cancer cases are positive for these oncoviruses. On the other hand, it has been recently pointed out that E6/E7 oncoproteins of high-risk HPV can upregulate the programmed cell death-1/programmed cell death-ligand 1 (PD-1/PD-L1) axis. Likewise, several recent reports showed that checkpoint blockades targeting PD-1/PD-L1 pathways have achieved efficient clinical responses via suppressing cancer progression and improving survival in several types of human cancers including metastatic cervical cancer. In this review, we summarize recent advances in our understanding of the PD-1/PD-L1 signaling pathway and its interaction with high-risk HPV and their oncoproteins, which could have an important impact on the management of HPV-associated cancers including cervical.

Keywords: high-risk HPV, oncoproteins, PD-1/PD-L1, cervical cancer, immunotherapy

\section{INTRODUCTION}

The World Health Organization (WHO) categorizes cervical cancer as the fourth most common cancer in females, accounting for $30 \%$ of cancer-mortality cases (1). In contrast, in developing countries, cervical cancer is classified as the most frequent gynecological cancer (2). The noted increase in disease prevalence in developing countries is partially attributed to immunecompromising conditions including HIV, which in turn is associated with a higher risk of persistent and multiple human papillomavirus (HPV) infections $(3,4)$. HPV is a non-enveloped doublestranded DNA virus that encompasses more than 150 types. On the basis of oncogenicity, HPVs are classified into high-risk $(16,18,31,33,35,39,45,51,52,56,58,59,68,69,82)$ and low-risk $(6,8,11,40,42,43,44,54,61,72)(5)$, of which high-risk HPV types 16 and 18 are the most widespread carcinogenic strains to humans. Additionally, results from case-control studies report a convincing association between high-risk HPV types 31, 33, 35, 39, 45, 51, 52, 56, 58, 59, and 66 
and carcinogenesis (5). To date, HPV, is the most common sexually transmitted viral infection worldwide; additionally, it is considered as the main causative agent of cervical intraepithelial neoplasia (CIN) and cervical cancer $(6,7)$.

The HPV DNA genome encodes for eight proteins, of which E5 and E6/E7 of high-risk HPV are reported oncogenes possessing transforming characteristics (8), while E5 oncoprotein affects cell alteration leading to carcinogenesis (9-11). More specifically, E5 oncoprotein functions by enhancing the expression of epidermal growth factor receptor-1 (EGFR-1) (12) and increases phosphorylation of protein kinase B (AKT), thereby activating the MEK/ERK1/2 pathway, thus inducing HPV-associated carcinogenesis $(13,14)$. Concomitantly, Yan et al. reported that in cervical cancer cells, inhibition of the PI3K/Akt pathway reduces epithelial-mesenchymal transition (EMT) as well as cellular invasion and migration, providing a potentially valuable therapeutic target for cervical cancer (15). On the other hand, gene transfer studies showed that in HPV16- and HPV18-induced cervical carcinoma, E6 and E7 are the chief viral oncoproteins responsible for cell cycle deregulation (16). The effects of E6 protein are mainly mediated through two pathways. Firstly, E6 serves as a ligand to $\mathrm{p} 53$, enhancing degradation via ubiquitin $(17,18)$. In healthy individuals or in HPV-negative cells, degradation of p53 is mediated through the ubiquitin-proteasome system (19). Secondly, E6 triggers telomerase activity contributing to the uncontrolled proliferative capacity of cancerous cells (20). Equally, E7 oncoprotein exerts its action by binding to several members of the retinoblastoma $(\mathrm{Rb})$ family of tumor suppressor proteins, mainly hypo-phosphorylated form of $\mathrm{pRb}$, resulting in destabilization of the $\mathrm{Rb} / \mathrm{E} 2 \mathrm{~F}$ complex, which in turn leads to an increase in gene transcription and initiate cell proliferation (21).

On the other hand, immune checkpoints are essential mechanisms that function under physiological conditions averting autoimmunity and minimizing allergic reactions (22). Programmed cell death-1 (PD-1) is a type I transmembrane protein receptor that upon interacting with its ligands, PDL1, and PD-L2, triggers a cascade of downstream signals and suppresses $\mathrm{T}$ cell activation (23). Since CD8+ T cells play a vital role in eliminating cancer cells, their inhibition interferes with cancer eradication and permits its immune escape (24). In cervical cancer, multiple studies have emphasized on the independent role of the PD-/PD-L1 pathway in cancerous cell proliferation and growth $(25,26)$ and the involvement of E5 and E6/E7 oncoproteins in the pathogenesis of cervical cancers $(27,28)$. However, the interrelation between high-risk HPV oncoproteins (E5, E6/E7) and PD1/PD-L1 pathways and their potential synergic effect are yet to be fully elucidated. Furthermore, identification of potential therapeutic options designed to target HPV-PD$1 / \mathrm{PD}-\mathrm{L} 1$ interaction in cervical cancers and CIN is essential given the prominence of HPV infections in this type of cancers. This review aims to underline the interrelation between high-risk HPV oncoproteins and PD-1/PD-L1 in the pathogenesis of cervical cancer, which could reinforce the role of PD-1/PD-L1 inhibitors as one of the main therapies for the management of HPV-positive human carcinomas including cervical.

\section{PD-1 AND PD-L1 PATHWAY}

As we mentioned above, the role of high-risk HPV in cervical carcinogenesis is well-established and documented $(29,30)$. On the other hand, the emergence of immunotherapy coupled with the exponential increase in understanding therapy-induced host immune response in hindering tumor proliferation played a pivotal role in the identification of novel immune checkpoint inhibitors for treatment of several types of cancers $(25,31,32)$. In physiological conditions, immune checkpoints are thought to be essential in regulating the immune system, thereby preventing the development of autoimmune diseases (33). In this context, the $\mathrm{PD}-1$ receptor is thought to be an immune checkpoint mediator, subduing tumor-induced immunity (34).

In healthy individuals, the PD-1 pathway regulates antigenmediated inflammatory response to ensure minimal damage to healthy tissues (35). Notably, in the presence of a recognizable antigen expressed by the major histocompatibility complex (MHC), an inflammatory response is triggered through the activation of $\mathrm{T}$ cells and recruitment of cytokine-producing cells (34). Immune tolerance is elicited following cytokineinduced PD-L1 expression in tissues and the activation of PD-1 protein on T-cells (34). Once activated, the PD-1/PD-L1 pathway inhibits T-cell receptor (TCR) signal transduction and CD28CD8 co-stimulation, thus hindering $\mathrm{T}$ cell activity (36). On the other side of the spectrum, toll-like receptors (TLR) function through sensing common pathogen features and activating innate immunity. Drug-induced activation of TLR9 was proven to moderately improve immunogenic tumor sensitivity and augment innate immunity to promote tumor regression, thus indicating plausible potential synergetic effects between TLR9 agonist and PD-1 inhibitors (37).

\section{PD-1 CHECKPOINTS AND CERVICAL CANCER}

Several studies showed an elevation of PD-L1 in a variety of cancers, including cervical. Immunohistochemistry studies were used to determine PD-L1 expression, and the staining intensity was used to quantify the levels of PD-L1 in tumor sections (38, 39). Elevated PD-L1 expression inhibits $\mathrm{T}$ cell activity and thereby favors a state of immune resistance (40). Further, a direct correlation between PD-L1 overexpression and poor overall survival in patients with cervical cancer was reported (41). Markedly, Wang et al. reported an overexpression of PD-L1 levels in CIN $1-2$ lesions $(20 / 21=95 \%)$ as opposed to histologically normal cervical epithelia (0/55) (42). On a similar note, Meng et al. and Feng et al., respectively, reported an overexpression of PD-L1 in more than $60 \%(59 / 97)$ and $45 \%(31 / 66)$ of patients with cervical cancer $(39,43)$. Additionally, PD-L1 expression was found in $34.4-96 \%$ of cervical carcinomas as opposed to histologically normal cervical tissues in which PD-L1 expression was minute $(26,44,45)$. Similarly, Reddy et al. reported 
an increase in PD-L1 expression by $29 \%$ in adenosquamous carcinomas, $17 \%$ in endocervical carcinomas, and $38 \%$ in squamous cell carcinomas (SCCs) (46). Furthermore, Heeren et al. found a higher abundance of PD-L1-positive immune cells in close proximity to metastatic tumors as compared with paired primary tumors. The quantification was done based on the presence or absence of PD-L1-positive tumor-infiltrating cells as well as the accumulation of immune cells around tumor fields forming a PD-L1-positive cordon. Additionally, the authors reported PD-L1-positive tumor-associated macrophages, expressing CD163, and/or CD14 (47). This implies the important role that $\mathrm{PD}-1 / \mathrm{PD}-\mathrm{L} 1$ axis plays in hindering immunity against cervical cancer. Remarkably, upregulated levels of PD-L1 in SCCs are associated with poorer disease-free and disease-specific survival rates in comparison to those with normal or minute PD-L1 levels (47). Moreover, presence of PD-L1-positive tumorassociated macrophages correlates with worst disease-specific survival rates in adenocarcinoma patients (47). Evidently, the $\mathrm{PD}-1 / \mathrm{PD}-\mathrm{L} 1$ pathway plays an important role in the pathogenesis of cervical cancer.

The illustrated PD-1 mechanism of action and the role it plays in the pathogenesis of a variety of cancers coupled with its unique structure-resembling that of cytotoxic T-lymphocyte-associated antigen 4 (CTLA-4) but with a unique ligand specificity and biological functionality-makes it an exceptional drug target (48). Unlike CTLA-4 ligands, earlier studies pointed out that PD1 ligands are selectively expressed in various cancers as well as within tumor microenvironments $(49,50)$; specifically, PD-1 was found to be quite prominent in various solid and hematological malignancies (49-54). An in vitro study by Fife et al. revealed that antibody-mediated inhibition of PD-L1 binding to PD-1 resulted in lower $\mathrm{T}$ cell motility and enhanced $\mathrm{T}$ cell-dendritic cell interaction (55). Together, these findings support the use of PD-1 inhibitors as a promising strategy for tumor immunotherapy.

Understanding the pathways through which PD-L1 checkpoint activation leads to the development and progression of solid tumors provides a path to investigate the effects of PD-L1 inhibitors on solid tumor regression. Phase 3 clinical trials revealed a statistically significant increase in overall survival in myeloma patients receiving nivolumab (PD-L1 inhibitor) with $73 \%$ overall survival as compared to $42 \%$ for those who received dacarbazine (standard treatment) (56). Administration of various doses of pembrolizumab in patients with recurrent metastatic cervical cancer showed an overall response rate (ORR) of $14.33-17 \%$ (57, 58). Similarly, in patients with recurrent or metastatic HPV-related cancers (19 cervical and five vaginal/vulvar carcinomas, CheckMate358 study, NCT02488759), administration of nivolumab showed an ORR of $26 \%$ in patients with cervical cancer (59). Notably, the response to nivolumab was unrelated to PD-L1 status or previous treatments. Thus, the use of PD-1 inhibitors for cervical cancer is a promising treatment strategy.

In this context, pembrolizumab, an immune checkpoint inhibitor, represents a full-length human IgG4/kappa monoclonal antibody that is directed against the $\mathrm{PD}-1$ protein $(60,61)$ and has been approved by the FDA as a secondline treatment for recurrent or metastatic carcinomas of the cervix, non-small cell lung, and urothelial as well as malignant melanoma (60). Pembrolizumab (Keytruda) was approved for the treatment of patients with recurrent and/or metastatic cervical cancer in 2018 based on the KEYNOTE 158 (NCT02628067) Phase II study which involved 98 patients with recurrent and/or metastatic cervical carcinomas (62). The objective response rate (ORR) among 77 patients was achieved in $14.3 \%$ including $2.6 \%$ complete responses and $11.7 \%$ patients having partial responses (62). Of note, the FDA also concurrently approved the PD-L1 immunohistochemistry 22C3 pharmDx test (Dako Agilent) as a companion diagnostic test to guide the patient selection process for pembrolizumab treatment (63). This is critically important since pembrolizumab as a single agent exhibits a limited efficacy in recurrent and/or metastatic setting in an unselected patient population (61). Moreover, an ongoing phase III trial (KEYNOTE-826 phase III trial, NCT03635567) aims to treat advanced or recurrent cervical cancer in the first line using pembrolizumab or a placebo plus one of four platinum- and taxane-based chemotherapy regimens (61). Notably, patients are being stratified based on PD-L1 expression (combined positive score $\geq 1$ ) by immunohistochemistry $(62,63)$.

Given that clinical benefits of pembrolizumab in cervical cancer are still sparse and limited, there is an unmet need for more trials and studies that explore the role of pembrolizumab in addition to other immune checkpoint inhibitors (e.g., PD1 (nivolumab and cemiplimab) and PD-L1 inhibitors (e.g., durvalumab, avelumab, and atezolizumab) (64). A combinatorial approach with immune checkpoint inhibitors is also warranted (65). This is particularly important given that immune suppression (impaired cellular response) caused by the activation of the inhibitory axis PD-1/PD-L1 strongly favors persistent HPV infections, viral integrations into the cervical epithelium, and concomitant expression of the key viral oncoproteins such as E6 and E7 proteins (64). In addition, a combined treatment of immune checkpoint inhibitors with other therapeutic modalities (e.g., bevacizumab, conventional chemotherapy, radiotherapy) is also a huge challenge.

\section{HPV ONCOPROTEINS AND PD-1/PD-L1 INTERACTION IN CERVICAL CANCER}

In the case of cervical cancer, high-risk HPVs are a determining factor in its pathogenesis; continual HPV infection is associated with pathogenesis of cervical cancer and is correlated with its prognosis. This, coupled with the significance of the PD-1/PD-L1 axis in cervical cancer etiology, has made it crucial to investigate the interrelation between E5 and E6/E7 oncoproteins and the PD-1/PD-L1 pathway in the pathogenesis of cervical cancer (Figure 1). Research has shown a significant association between HPV positivity and enhanced PD-L1 expression $(9,42,66)$. While studies highlighting the association between E5 oncoprotein of high-risk HPV and PD-1/PD-L1 expression in cervical cancer are scarce, Kim et al. investigated the effects of E5 expression on epidermal growth factor receptor-1 (EGFR1) and vascular endothelial growth factor (VEGF) in cervical cancer cell lines (12), concluding that E5 oncoprotein activates EGFR1 thereby 


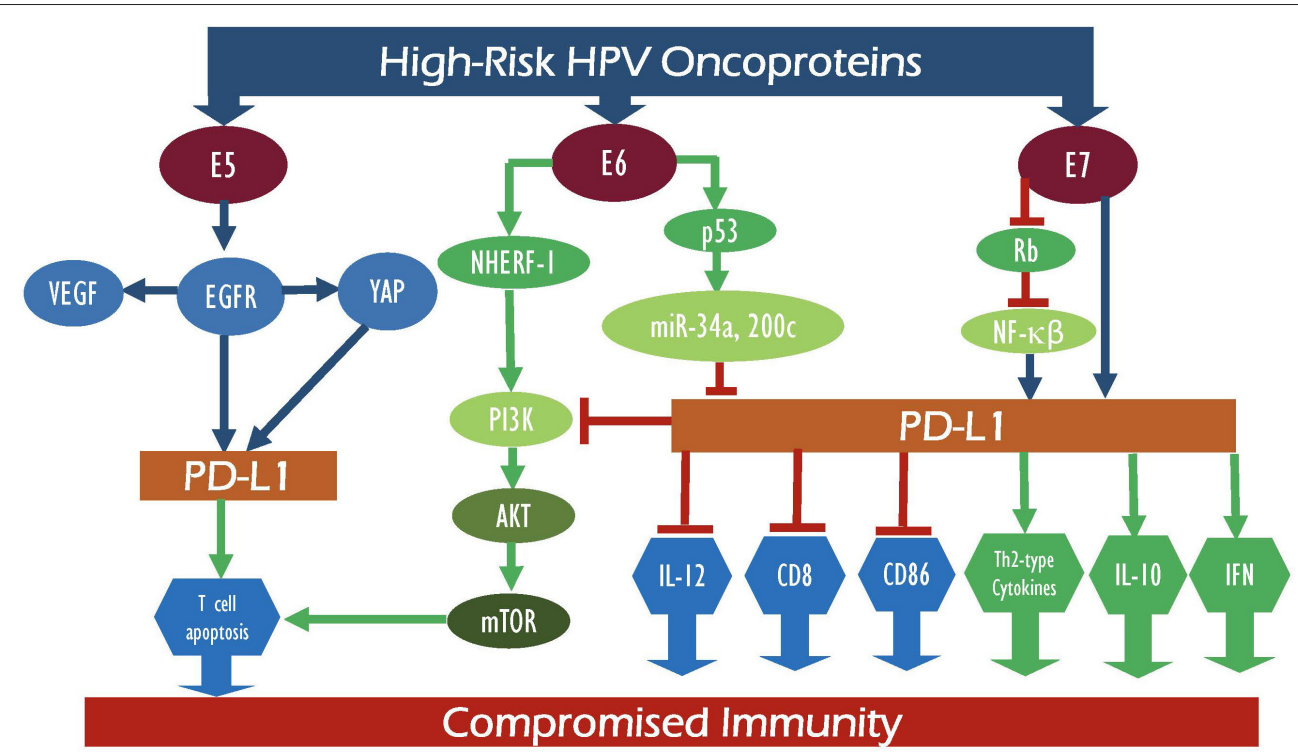

FIGURE 1 | Schematic outline showing the potential interaction between E5, E6, and E7 of high-risk HPV oncoproteins with their intermediate proteins as well as their signaling pathways which can deregulate PD-1 and PD-L1 expression patterns, consequently leading to compromised immunity.

upregulating the expression of VEGF (9). Furthermore, HPV16E6 binds to $\mathrm{Na}^{+} / \mathrm{H}^{+}$exchanger regulatory factor-1 (NHERF-1) and results in the breakdown of NHERF-1 via the proteasome pathway (67). Also, E7 combines with E6 to underpin NHERF1 degradation, thus triggering the PI3K/AKT pathway (68). Moreover, in vitro data link loss of NHERF-1 in cervical cancer with increase in cellular growth, proliferation, cell cycle, and ERK signaling stimulated by EGFR $(69,70)$. Incessant EGFR activation by NHERF-1 correlates with poor prognosis in cervical cancer (70). Also, NHERF-1 has been found to play a role in cisplatin resistance of cervical cancer cells by inhibiting AKT and ERK signaling pathways (71). Moreover, loss of NHERF-1 expression in the cervical cell line promoted cellular adhesion, wound healing, and invasion, indicating NHERF-1 as a plausible therapeutic target for cervical cancer patients (72). Similarly, $\mathrm{He}$ et al. report that EGFR activation increases the expression of yes-associated protein (YAP), thus inducing cervical cancer cell proliferation and migration (73). More importantly, Chen et al. investigated the effects of EGFR1 on PD-L1 activity in NSCLC (74). This study found that EGFR1 activation induces PD-L1 expression through the phosphorylation of ERK1/2/cJun, potentially causing $\mathrm{T}$ cell apoptosis. On the other hand, Lee et al. analyzed the role YAP plays in the activation of the PD-1/PD-L1 pathway (75). The study concluded that YAP regulates the transcription of $\mathrm{PD}-\mathrm{L} 1$; thus, targeting $\mathrm{PD}-\mathrm{L} 1$ either directly or through YAP could be a potential therapeutic target (75). Yang et al. showed a correlation between increased PD1 expression on T cells and persistent high-risk HPV infection with the development of cervical intraepithelial neoplasia (CIN) (44). Altogether, E5 oncoprotein activates EGFR, which enhances YAP leading to the upregulation of PD-L1, thus initiating T cell apoptosis and persistent HPV infections and augmenting the risk of cervical cancer development $(44,73,75)$. MicroRNAs
(miRs), a class of small regulatory, non-coding RNA molecules, play a vital role in gene expression modulation and regulating major pathways (76). High-risk HPV oncoproteins interact with miRs to regulate the onset and progression of cervical cancer (77). One such miR, miR-34a, is directly regulated by p53 and targets molecules regulating cell proliferation, cellular apoptosis, G1 arrest, DNA repair, and senescence (78-81). HPV-E6 blocks miR-34a expression via the 553 pathway inducing virus-infected cell survival and cancer cell proliferation and metastasis $(82,83)$. A study by Li et al. (81) showed loss of miR-34a expression in precancerous cervical lesions, indicating this as an early-onset event in cervical cancer development (81). Several studies have shown reduced miR-34a expression in both cervical cancer cells and tissues associated with invasive carcinomas (81, 82, 84-86). Moreover, Cortez et al. (87) and Wang et al. (88) analyzed the role of miR-24a in controlling PD-L1 activity (87). In vitro and in vivo data showed that $\mathrm{p} 53$ regulates $\mathrm{PD}-\mathrm{L} 1$ via miR-34a $(87,88)$. miR-34a directly attaches to the PD-L1 3 -untranslated region, inhibiting PD-L1; thus, induction of miR-34a resulted in reduced PD-L1-specific $\mathrm{T}$ cell apoptosis, indicating that tumor immune evasion is regulated by the p53/miR-34a/PDL1 axis $(87,88)$. Expression of another $\mathrm{miR}, \mathrm{miR}-200 \mathrm{c}$, was lost in cervical cancer tissue samples and cell lines (89). Elevated p53 levels inhibit miR200c levels and correlate with increased cervical cell invasion, migration, and proliferation abilities (90, 91). Further, miR-200c expression is significantly reduced in PD-L1-positive samples, suggesting miR-200c as a potential regulator of PD-L1 (92). Thus, increased PD-L1 expression can elevate miR-200c expression, inhibiting tumor cell migration, and metastasis (93).

Moreover, a study by Liu et al. investigated the mechanisms underlying HPV-induced evasion of cervical cancer cells from the host immune system via the PD-L1/PD-1 signaling pathway (66). Quantification of HPV16-E7 expression using 
immunohistochemistry and RT-PCR analyses revealed low levels of HPV16-E7 in normal tissues in comparison to elevated levels detected in cervical cancer tissues; immunohistochemical analysis showed strong PD-L1 expression in cervical cancer samples $(P=0.017)(66)$. A statistically significant $(P=0.043)$ positive correlation between HPV16-E7 and PD-L1 protein expression in cervical cancer tissues was reported, indicating that HPV16-E7 potentially downregulates lymphocyte proliferation by stimulating the PD-L1 pathway and weakening the immune response to tumor cells (66). Also, the association of HPV-E7 to $\mathrm{pRb}$ results in the degradation of the cell cycle allowing cells to undergo unchecked proliferation $(94,95)$. E7 also binds to and

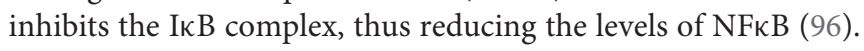
HPV16-E6 and E7 proteins repress basal and TNF- $\alpha$-inducible NF-kB activity in cervical cancer cells, promoting onset of cervical cancer (97). Loss of NF-kB allows the virus to restrict the host immune response and stimulates continuous HPV infection which can in turn trigger clonal growth and immortalization of cervical epithelial cells (97). Furthermore, PD-L1 expression is regulated by NF- $\mathrm{B}$ during EMT signaling; NF- $\kappa \mathrm{B}$ activates PD-L1, promoting cancer growth, and progression (98). Herein it is important to highlight that the interaction between PDL1 and HPV oncoproteins could be specific to only certain types of high-risk HPVs or coinfection with more than one type since more than $96 \%$ of human cervical cancers are positive for HPVs, while around $40 \%$ of these cancer cases overexpress PD-L1, as reported by Meng et al. (39) and Feng et al. (43).

Furthermore, numerous studies investigated the role of PD-L1 expression in the prognosis and therapeutic efficacy of cervical cancer patients. Yang et al. explored the correlation between the modulation of the PD-1/PD-L1 axis and CIN grading of cervical cells in high-risk HPV (-) and (+) women (44). In patients with high-risk HPV, ELISA revealed a reduced expression of Th1 cytokines, interferon-c (IFN-c), and interleukin-12 (IL12). In contrast, Th2-type cytokine and interleukin-10 (IL-10) were increased as compared with high-risk (-) HPV. Highrisk HPV positivity correlated with increasing CIN grade. As for the PD-1/PD-L1 axis, Yang et al. showed that the activation of the PD-1/PD-L1 pathway suppresses cell-mediated immunity (CMI), by inhibiting pro-inflammatory cytokines and upregulating anti-inflammatory cytokines, thus leading to localized immunosuppression and high-risk HPV-induced CIN progression (44) (Figure 1). A study by Qin et al. (99) showed that HPV-induced somatic mutations played a vital role in the inhibitory tumor microenvironment resulting in aberrant expression of checkpoint-related genes (CTLA-4, PD-1, and PDL1) (99). Furthermore, the expression of HPV oncoproteinassociated master regulators was determined and their effect on the functionality of the immune system in cervical cancer was analyzed (99); thus, a positive correlation $(p<0.001)$ was reported between certain HPV-induced master regulators, specifically ENO1, FOSB, PA2G4, SOX9, TEAD4, FOXO4, and MNT, in cervical cancer samples $(n=306)$ in comparison with normal cervix $(n=11)$ tissue obtained from The Cancer Genome Atlas (TCGA). This proposes the induction of an immune-deficient state caused by the HPVE6/E7 expression that is mediated through a variety of pathways including the PD1/PD-L1 axis (99). In summary, a positive correlation between HPV E5, E6, and E7 oncoproteins and enhanced PD-1/PDL1 expression has been reported with tumor metastasis (22), tumor progression (100), and poor prognosis (47) in cervical cancer. However, the exact underlying mechanism by which these two elements are interconnected still remains nascent. Studies suggest that E6/E7 activates the PD-1/PD-L1 axis causing an increase in Th2-type cytokine and IL-10 expression and a decrease in Th1 cytokine IFN-c and IL-12 expression leading to immunosuppression and further progression of CIN $(101,102)$ (Figure 1).

To identify therapeutic targets for local immune modulation, multiparameter flow cytometric T-cell profiling of primary cervical tumors (PT) and tumor-draining lymph nodes (TDLN) of cervical cancer was performed (103). The study reported that the inhibition of PD-1 led to a significant increase in E6-specific T-cell responses in $80 \%$ of HPV16+ TDLN and in $20 \%$ of HPV16+ PT as well as enhanced expression levels of $\mathrm{CD} 8+$, FoxP3+, and $\mathrm{CD} 25+\mathrm{T}$ cells, thus confirming its efficacy (103). Taking into account the promising results reported from $\mathrm{PD}-1 / \mathrm{PD}-\mathrm{L} 1$ inhibitors in the treatment of high-risk HPV-mediated cervical cancers (104), the efforts are now directed toward combination therapy with PD-1 pathway blockers and E6/E7-targeted therapy. Effects of CRISPR/Cas9 to target both $\mathrm{HPV}$ and $\mathrm{PD}-1$ in vitro and in vivo models (104) were tested, and results showed that the combination therapy with gRNA-PD-1 and gRNA-HPV16 E6/E7 significantly reduced tumor growth and enhanced survival. Moreover, an increase in the population of dendritic cells, CD8+, and CD4+ $\mathrm{T}$ lymphocyte was observed following the administration of the combination therapy (104). Ongoing clinical trials are investigating the use of various $\mathrm{PD}-1$ inhibitors/modulators [nivolumab (105), atezolizumab (106), and pembrolizumab (107)] in combination with concurrent chemoradiation therapy (CCRT) for the management of advanced and recurrent cervical cancers. Additionally, promising results from a phase II clinical trial investigating the effects of combined immune checkpoint inhibitor and tumor-specific vaccine (ISA 101, a synthetic longpeptide HPV-16 vaccine) for patients with incurable HPV-16induced cancer was obtained (108).

These investigations show that PD-1/PD-L1 inhibitors could be in fact promising therapeutic targets for HPV-associated cancers, especially cervical. Although studies analyzing the potency of $\mathrm{PD}-1 / \mathrm{PD}-\mathrm{L} 1$ inhibitors in the management of cervical cancer are rare, there are few studies on the clinical application of PD-1/PD-L1 inhibitors and/or new inhibitors targeting other elements of cell death program. Given the promising results of these preliminary studies, we believe more investigations are necessary to elucidate the role and efficacy of such treatments. The major limitations in studies conducted thus far include lack of data regarding overall survival, drug resistance, and the underlying mechanisms. Such data can aid in selecting patients for cell death program inhibitors, including PD-1/PD-L1, and pave the way for new therapeutic interventions. On the other hand, it is important to highlight that PD-1/PD-L1 inhibitors have proven to be a viable therapy that can reactivate immune 
response against cancerous cells and favor apoptosis as opposed to tumor growth and proliferation. Moreover, current clinical trials investigating the use of cell death program inhibitors, including $\mathrm{PD}-1 / \mathrm{PD}-\mathrm{L} 1$, for the treatment of cervical cancer are limited to advanced, persistent, and metastatic cancers. Therefore, it would be interesting to investigate the effectiveness of these agents on early-stage cervical cancers in a small cohort. Additionally, the promising outcomes noted in combination therapy of PD-1 inhibitors with other treatment modalities in various types of cancers favor combination therapy as opposed to PD-1 inhibitor monotherapy for cervical cancer management.

\section{CONCLUSION}

It is known that the majority of human cervical cancers are positive for high-risk HPVs that involve three known oncoproteins, E5, E6, and E7, which regulate cell-cycle and tumor-suppressor genes, thereby affecting apoptosis and cell death program. We have reviewed the cross talk between highrisk HPV oncoproteins and the PD-1/PD-L1 pathway in the pathogenesis of cervical cancer. It is evident that there is a strong interplay between oncoproteins of high-risk HPV and cell death, including PD-1 activation, as reported in several investigations. More specifically, HPV oncoproteins E5 and E6/E7 can activate the $\mathrm{PD}-1 / \mathrm{PD}-\mathrm{L} 1$ axis; hence, further research is required to

\section{REFERENCES}

1. WHO. Cervical Cancer. WHO. (2018) Available online at: https://www.who. int/cancer/prevention/diagnosis-screening/cervical-cancer/en/ (accessed April 02, 2020).

2. Bruni L, Albero G, Serrano B, Mena M, Gómez D, Muñoz J, et al. ICO/IARC Information Centre on HPV and Cancer (HPV Information Centre). Human Papillomavirus and Related Diseases in report. Barcelona: ICO/IARC (2018).

3. Clifford GM, Tully S, Franceschi S. Carcinogenicity of human papillomavirus (HPV) types in HIV-positive women: a meta-analysis from HPV infection to cervical cancer. Clin Infect Dis. (2017) 64:1228-35. doi: 10.1093/cid/cix135

4. Arbyn M, Weiderpass E, Bruni L, de Sanjosé S, Saraiya M, Ferlay J, et al. Estimates of incidence and mortality of cervical cancer in 2018: a worldwide analysis. Lancet Glob. Health. (2020) 8:e191-203. doi: 10.1016/S2214-109X1930482-6

5. Bouvard V, Baan R, Straif K, Grosse Y, Secretan B, El Ghissassi F, et al. A review of human carcinogens-part B: biological agents. Lancet Oncol. (2009) 10:321-2. doi: 10.1016/S1470-20450970096-8

6. Fang J, Zhang H, Jin S. Epigenetics and cervical cancer: from pathogenesis to therapy. Tumour Biol. (2014) 35:5083-93. doi: 10.1007/s13277-014-1737-z

7. Bhat P, Bergot A-S, Waterhouse N, Frazer IH. Human papillomavirus E7 oncoprotein expression by keratinocytes alters the cytotoxic mechanisms used by CD8 T cells. Oncotarget. (2017) 9:6015-27. doi: 10.18632/oncotarget.23210

8. Yeo-Teh NSL, Ito Y, Jha S. High-risk human papillomaviral oncogenes E6 and E7 target key cellular pathways to achieve oncogenesis. Int J Mol Sci. (2018) 19:1706. doi: 10.3390/ijms19061706

9. Kim SH, Juhnn YS, Kang S, Park SW, Sung MW, Bang YJ, et al. Human papillomavirus 16 E5 up-regulates the expression of vascular endothelial growth factor through the activation of epidermal growth factor receptor, MEK/ ERK1,2 and PI3K/Akt. Cell Mol Life Sci. (2006) 63:930-8. doi: 10.1007/s00018-005-5561-x

10. Suprynowicz FA, Disbrow GL, Krawczyk E, Simic V, Lantzky K, Schlegel R. HPV-16 E5 oncoprotein upregulates lipid raft components caveolin-1 and elucidate the association between HPV status and the efficacy of PD-1/PD-L1 inhibitors and/or other inhibitors related to cell death program in cervical cancer. On the other hand, analyzing the interaction between oncoproteins of high-risk HPVs and cell death program including PD-1/PD-L1 can also help in identifying candidate novel biomarkers that can help in the prognosis of cervical cancer patients.

\section{AUTHOR CONTRIBUTIONS}

All authors have read and agreed to the published version of the manuscript.

\section{FUNDING}

A-EA lab was supported by grants from Qatar University: QUHICMED-19/20-1, GCC \# 2017-002 QU/KU, and QUST-1-CMED2020-2.

\section{ACKNOWLEDGMENTS}

We would like to thank Mrs. A. Kassab for her critical reading of the manuscript. The publication of this article is funded by the Qatar National Library.

ganglioside GM1 at the plasma membrane of cervical cells. Oncogene. (2008) 27:1071-8. doi: 10.1038/sj.onc. 1210725

11. Oh JM, Kim SH, Cho EA, Song YS, Kim WH, Juhnn YS. Human papillomavirus type 16 E5 protein inhibits hydrogen peroxide-induced apoptosis by stimulating ubiquitin-proteasome-mediated degradation of Bax in human cervical cancer cells. Carcinogenesis. (2009) 31:402-10. doi: 10.1093/carcin/bgp318

12. Pim D, Collins M, Banks L. Human papillomavirus type 16 E5 gene stimulates the transforming activity of the epidermal growth factor receptor. Oncogene. (1992) 7:27-32.

13. Crusius K, Kaszkin M, Kinzel V, Alonso A. The human papillomavirus type 16 E5 protein modulates phospholipase C-gamma-1 activity and phosphatidyl inositol turnover in mouse fibroblasts. Oncogene. (1999) 18:6714-8. doi: 10.1038/sj.onc. 1203075

14. Zhang L, Wu J, Ling MT, Zhao L, Zhao K-N. The role of the PI3K/Akt/mTOR signalling pathway in human cancers induced by infection with human papillomaviruses. Mol Cancer. (2015) 14:87. doi: 10.1186/s12943-0150361-x

15. Yan S-P, Chu D-X, Qiu H-F, Xie Y, Wang C-F, Zhang J-Y, et al. LncRNA LINC01305 silencing inhibits cell epithelial-mesenchymal transition in cervical cancer by inhibiting TNXB-mediated PI3K/Akt signalling pathway. J Cell Mol Med. (2019) 23:2656-66. doi: 10.1111/jcmm.14161

16. zur Hausen $\mathrm{H}$. Immortalization of human cells and their malignant conversion by high risk human papillomavirus genotypes. Semin Cancer Biol. (1999) 9:405-11. doi: 10.1006/scbi.1999.0144

17. Scheffner M, Werness BA, Huibregtse JM, Levine AJ, Howley PM. The E6 oncoprotein encoded by human papillomavirus types 16 and 18 promotes the degradation of p53. Cell. (1990) 63:1129-36. doi: 10.1016/0092-8674(90)90409-8

18. Werness BA, Levine AJ, Howley PM. Association of human papillomavirus types 16 and 18 E6 proteins with p53. Science. (1990) 248:76-9. doi: $10.1126 /$ science. 2157286

19. Momand J, Zambetti GP, Olson DC, George D, Levine AJ. The $\mathrm{mdm}-2$ oncogene product forms a complex with the p53 protein 
and inhibits p53-mediated transactivation. Cell. (1992) 69:1237-45. doi: 10.1016/0092-8674(92)90644-R

20. Oh ST, Kyo S, Laimins LA. Telomerase activation by human papillomavirus type 16 E6 protein: induction of human telomerase reverse transcriptase expression through Myc and GC-rich Sp1 binding sites. J Virol. (2001) 75:5559-66. doi: 10.1128/JVI.75.12.5559-5566.2001

21. Münger K, Phelps WC, Bubb V, Howley PM, Schlegel R. The E6 and E7 genes of the human papillomavirus type 16 together are necessary and sufficient for transformation of primary human keratinocytes. J Virol. (1989) 63:4417-21. doi: 10.1128/JVI.63.10.4417-4421.1989

22. Yang W, Lu YP, Yang YZ, Kang JR, Jin YD, Wang HW. Expressions of programmed death (PD)-1 and PD-1 ligand (PD-L1) in cervical intraepithelial neoplasia and cervical squamous cell carcinomas are of prognostic value and associated with human papillomavirus status. J Obstet Gynaecol Res. (2017) 43:1602-12. doi: 10.1111/jog.13411

23. Dong $\mathrm{Y}$, Sun $\mathrm{Q}$, Zhang $\mathrm{X}$. PD-1 and its ligands are important immune checkpoints in cancer. Oncotarget. (2017) 8:2171-86. doi: 10.18632/oncotarget.13895

24. Lu D, Ni Z, Liu X, Feng S, Dong X, Shi X, et al. Beyond T cells: understanding the role of PD-1/PD-L1 in tumor-associated macrophages. J Immunol Res. (2019) 2019:1919082. doi: 10.1155/2019/1919082

25. Alsaab HO, Sau S, Alzhrani R, Tatiparti K, Bhise K, Kashaw SK, et al. PD1 and PD-L1 checkpoint signaling inhibition for cancer immunotherapy: mechanism, combinations, and clinical outcome. Front Pharmacol. (2017) 8:561. doi: 10.3389/fphar.2017.00561

26. Enwere EK, Kornaga EN, Dean M, Koulis TA, Phan T, Kalantarian M, et al. Expression of PD-L1 and presence of CD8-positive T cells in pre-treatment specimens of locally advanced cervical cancer. Mod Pathol. (2017) 30:577-86. doi: 10.1038/modpathol.2016.221

27. Liu C, Lin J, Li L, Zhang Y, Chen W, Cao Z, et al. HPV16 early gene E5 specifically reduces miRNA-196a in cervical cancer cells. Sci Rep. (2015) 5:7653. doi: $10.1038 /$ srep 07653

28. Cancer Genome Atlas Research N, Albert Einstein College of Medicine M, Analytical Biological S, Barretos Cancer H, Baylor College of Medicine M, Beckman Research Institute of City of Hope H, et al. Integrated genomic and molecular characterization of cervical cancer. Nature. (2017) 543:378-84. doi: 10.1038/nature21386

29. Parkin DM, Bray F. Chapter 2: the burden of HPV-related cancers. Vaccine. (2006) 24 (Suppl.3):S3/11-25. doi: 10.1016/j.vaccine.2006.05.111

30. Al Moustafa A-E, Al-Awadhi R, Missaoui N, Adam I, Durusoy R, Ghabreau $\mathrm{L}$, et al. Human papillomaviruses-related cancers. Presence and prevention strategies in the Middle east and north African regions. Hum Vaccin Immunother. (2014) 10:1812-21. doi: 10.4161/hv.28742

31. Liu Y, Wu L, Tong R, Yang F, Yin L, Li M, et al. PD-1/PDL1 inhibitors in cervical cancer. Front Pharmacol. (2019) 10:65. doi: 10.3389/fphar.2019.00065

32. Wang Y, Li G. PD-1/PD-L1 blockade in cervical cancer: current studies and perspectives. Front Med. (2019) 13:438-50. doi: 10.1007/s11684-018-0674-4

33. Sharpe AH, Wherry EJ, Ahmed R, Freeman GJ. The function of programmed cell death 1 and its ligands in regulating autoimmunity and infection. Nat Immunol. (2007) 8:239-45. doi: 10.1038/ni1443

34. Mahoney KM, Rennert PD, Freeman GJ. Combination cancer immunotherapy and new immunomodulatory targets. Nat Rev Drug Discov. (2015) 14:561-84. doi: 10.1038/nrd4591

35. Francisco LM, Sage PT, Sharpe AH. The PD-1 pathway in tolerance and autoimmunity. Immunol Rev. (2010) 236:219-42. doi: 10.1111/j.1600-065X.2010.00923.x

36. Riley JL. PD-1 signaling in primary T cells. Immunol Rev. (2009) 229:114-25. doi: 10.1111/j.1600-065X.2009.00767.x

37. Reilley MJ, Morrow B, Ager CR, Liu A, Hong DS, Curran MA. TLR9 activation cooperates with $\mathrm{T}$ cell checkpoint blockade to regress poorly immunogenic melanoma. J Immunother Cancer. (2019) 7:323. doi: 10.1186/s40425-019-0811-x

38. Wang X, Teng F, Kong L, Yu J. PD-L1 expression in human cancers and its association with clinical outcomes. Onco Targets Ther. (2016) 9:5023-39. doi: 10.2147/OTT.S105862

39. Meng Y, Liang $\mathrm{H}, \mathrm{Hu}$ J, Liu S, Hao X, Wong MSK, et al. PD-L1 expression correlates with tumor infiltrating lymphocytes and response to neoadjuvant chemotherapy in cervical cancer. J Cancer. (2018) 9:2938-45. doi: $10.7150 /$ jca. 22532

40. Piersma SJ. Immunosuppressive tumor microenvironment in cervical cancer patients. Cancer Microenviron. (2011) 4:361-75. doi: 10.1007/s12307-011-0066-7

41. Gu X, Dong M, Liu Z, Mi Y, Yang J, Zhang Z, et al. Elevated PD-L1 expression predicts poor survival outcomes in patients with cervical cancer. Cancer Cell Int. (2019) 19:146. doi: 10.1186/s12935-019-0861-7

42. Mezache L, Paniccia B, Nyinawabera A, Nuovo GJ. Enhanced expression of PD L1 in cervical intraepithelial neoplasia and cervical cancers. Mod Pathol. (2015) 28:1594-602. doi: 10.1038/modpathol.2015.108

43. Feng YC, Ji WL, Yue N, Huang YC, Ma XM. The relationship between the PD-1/PD-L1 pathway and DNA mismatch repair in cervical cancer and its clinical significance. Cancer Manag Res. (2018) 10:105-13. doi: 10.2147/CMAR.S152232

44. Yang W, Song Y, Lu YL, Sun JZ, Wang HW. Increased expression of programmed death (PD)-1 and its ligand PD-L1 correlates with impaired cell-mediated immunity in high-risk human papillomavirusrelated cervical intraepithelial neoplasia. Immunology. (2013) 139:513-22. doi: $10.1111 / \mathrm{imm} .12101$

45. Chen Z, Pang N, Du R, Zhu Y, Fan L, Cai D, et al. Elevated expression of programmed death-1 and programmed death ligand-1 negatively regulates immune response against cervical cancer cells. Mediators Inflamm. (2016) 2016:6891482. doi: 10.1155/2016/6891482

46. Reddy OL, Shintaku PI, Moatamed NA. Programmed death-ligand 1 (PDL1) is expressed in a significant number of the uterine cervical carcinomas. Diagn Pathol. (2017) 12:45. doi: 10.1186/s13000-017-0631-6

47. Heeren AM, Punt S, Bleeker MCG, Gaarenstroom KN, van der Velden J, Kenter GG, et al. Prognostic effect of different PD-L1 expression patterns in squamous cell carcinoma and adenocarcinoma of the cervix. Mod Pathol. (2016) 29:753-63. doi: 10.1038/modpathol.2016.64

48. Ramagopal UA, Liu W, Garrett-Thomson SC, Bonanno JB, Yan Q, Srinivasan $\mathrm{M}$, et al. Structural basis for cancer immunotherapy by the first-in-class checkpoint inhibitor ipilimumab. Proc Natl Acad Sci USA. (2017) 114:E422332. doi: 10.1073/pnas.1617941114

49. Curiel TJ, Wei S, Dong H, Alvarez X, Cheng P, Mottram P, et al. Blockade of B7-H1 improves myeloid dendritic cell-mediated antitumor immunity. Nat Med. (2003) 9:562-7. doi: 10.1038/nm863

50. Brahmer JR, Tykodi SS, Chow LQ, Hwu WJ, Topalian SL, Hwu P, et al. Safety and activity of anti-PD-L1 antibody in patients with advanced cancer. $N$ Engl J Med. (2012) 366:2455-65. doi: 10.1056/NEJMoa1200694

51. Vranic S, Ghosh N, Kimbrough J, Bilalovic N, Bender R, Arguello D, et al. PD-L1 status in refractory lymphomas. PLoS ONE. (2016) 11:e0166266. doi: 10.1371/journal.pone.0166266

52. Goodman A, Patel SP, Kurzrock R. PD-1-PD-L1 immune-checkpoint blockade in B-cell lymphomas. Nat Rev Clin Oncol. (2017) 14:203-20. doi: 10.1038/nrclinonc.2016.168

53. Xu-Monette ZY, Zhou J, Young KH. PD-1 expression and clinical PD-1 blockade in B-cell lymphomas. Blood. (2018) 131:68-83. doi: 10.1182/blood-2017-07-740993

54. Noh BJ, Kwak JY, Eom DW. Immune classification for the PD-L1 expression and tumour-infiltrating lymphocytes in colorectal adenocarcinoma. $B M C$ Cancer. (2020) 20:58-58. doi: 10.1186/s12885-020-6553-9

55. Fife BT, Pauken KE, Eagar TN, Obu T, Wu J, Tang Q, et al. Interactions between PD-1 and PD-L1 promote tolerance by blocking the TCR-induced stop signal. Nat Immunol. (2009) 10:1185-92. doi: 10.1038/ni.1790

56. Sunshine J, Taube JM. PD-1/PD-L1 inhibitors. Curr Opin Pharmacol. (2015) 23:32-8. doi: 10.1016/j.coph.2015.05.011

57. Frenel JS, Le Tourneau C, O’Neil B, Ott PA, Piha-Paul SA, Gomez-Roca C, et al. Safety and efficacy of pembrolizumab in advanced, programmed death ligand 1-positive cervical cancer: results from the phase Ib KEYNOTE-028 trial. J Clin Oncol. (2017) 35:4035-41. doi: 10.1200/JCO.2017.74.5471

58. Schellens JHM, Marabelle A, Zeigenfuss S, Ding J, Pruitt SK, Chung HC. Pembrolizumab for previously treated advanced cervical squamous cell cancer: preliminary results from the phase 2 KEYNOTE-158 study. J Clin Oncol. (2017) 35(15 Suppl.):5514. doi: 10.1200/JCO.2017.35.15_suppl.5514

59. Hollebecque A, Meyer T, Moore KN, Machiels JPH, Greve JD, López-Picazo JM, et al. An open-label, multicohort, phase I/II study of nivolumab in 
patients with virus-associated tumors (checkmate 358): efficacy and safety in recurrent or metastatic (R/M) cervical, vaginal, and vulvar cancers. J Clin Oncol. (2017) 35(15 Suppl.):5504. doi: 10.1200/JCO.2017.35.15_suppl.5504

60. du Rusquec P, de Calbiac O, Robert M, Campone M, Frenel, JS. Clinical utility of pembrolizumab in the management of advanced solid tumors: an evidence-based review on the emerging new data. Cancer Manag Res. (2019) 11:4297-312. doi: 10.2147/CMAR.S151023

61. Marret G, Borcoman E, Le Tourneau C. Pembrolizumab for the treatment of cervical cancer. Expert Opin Biol Ther. (2019) 19:871-7. doi: 10.1080/14712598.2019.1646721

62. Chung HC, Ros W, Delord JP, Perets R, Italiano A, Shapira-Frommer R, et al. Efficacy and safety of pembrolizumab in previously treated advanced cervical cancer: results from the phase II KEYNOTE-158 study. J Clin Oncol. (2019) 37:1470-8. doi: 10.1200/JCO.18.01265

63. Vranic S, Arguello D, Contreras E, Cimic A, Gatalica Z. 1057PMolecular profiling reveals novel targetable biomarkers in neuroendocrine carcinoma of the uterine cervix. Ann Oncol. (2019) 30(Suppl.5):65. doi: 10.1093/annonc/mdz250.065

64. Minion LE, Tewari KS. Cervical cancer - state of the science: from angiogenesis blockade to checkpoint inhibition. Gynecol Oncol. (2018) 148:609-21. doi: 10.1016/j.ygyno.2018.01.009

65. Rubinstein MM, Makker V. Optimizing immunotherapy for gynecologic cancers. Curr Opin Obstet Gynecol. (2020) 32:1-8. doi: $10.1097 / G C O .0000000000000603$

66. Liu C, Lu J, Tian H, Du W, Zhao L, Feng J, et al. Increased expression of PDL1 by the human papillomavirus $16 \mathrm{E} 7$ oncoprotein inhibits anticancer immunity. Mol Med Rep. (2017) 15:1063-70. doi: 10.3892/mmr.2017.6102

67. Drews CM, Case S, Vande Pol SB. E6 proteins from high-risk HPV, lowrisk HPV, and animal papillomaviruses activate the Wnt/ $\beta$-catenin pathway through E6AP-dependent degradation of NHERF1. PLoS Pathog. (2019) 15:e1007575. doi: 10.1371/journal.ppat.1007575

68. Pim D, Bergant M, Boon SS, Ganti K, Kranjec C, Massimi P, et al. Human papillomaviruses and the specificity of PDZ domain targeting. FEBS J. (2012) 279:3530-7. doi: 10.1111/j.1742-4658.2012.08709.x

69. Accardi R, Rubino R, Scalise M, Gheit T, Shahzad N, Thomas M, et al. E6 and E7 from human papillomavirus type 16 cooperate to target the $\mathrm{PDZ}$ protein Na/H exchange regulatory factor 1. J Virol. (2011) 85:8208-16. doi: 10.1128/JVI.00114-11

70. Peng Z, Wang Q, Zhang Y, He J, Zheng J. EBP50 interacts with EGFR and regulates EGFR signaling to affect the prognosis of cervical cancer patients. Int J Oncol. (2016) 49:1737-45. doi: 10.3892/ijo.2016.3655

71. Tao T, Yang X, Qin Q, Shi W, Wang Q, Yang Y, et al. NHERF1 enhances cisplatin sensitivity in human cervical cancer cells. Int J Mol Sci. (2017) 18:5. doi: 10.3390/ijms18010005

72. Wang L, Qi Y, Xiong Y, Peng Z, Ma Q, Zhang Y, et al. Ezrin-radixin-moesin binding phosphoprotein 50 (EBP50) suppresses the metastasis of breast cancer and HeLa cells by inhibiting matrix metalloproteinase- 2 activity. Anticancer Res. (2017) 37:4353-60. doi: 10.21873/anticanres.11829

73. He C, Mao D, Hua G, Lv X, Chen X, Angeletti PC, et al. The Hippo/YAP pathway interacts with EGFR signaling and HPV oncoproteins to regulate cervical cancer progression. EMBO Mol Med. (2015) 7:1426-49. doi: $10.15252 / \mathrm{emmm} .201404976$

74. Chen N, Fang W, Zhan J, Hong S, Tang Y, Kang S, et al. Upregulation of PD-L1 by EGFR activation mediates the immune escape in EGFRdriven NSCLC: implication for optional immune targeted therapy for NSCLC patients with EGFR mutation. J Thorac Oncol. (2015) 10:910-23. doi: 10.1097/JTO.0000000000000500

75. Lee BS, Park DI, Lee DH, Lee JE, Yeo MK, Park YH, et al. Hippo effector YAP directly regulates the expression of PD-L1 transcripts in EGFR-TKI-resistant lung adenocarcinoma. Biochem Biophys Res Commun. (2017) 491:493-9. doi: 10.1016/j.bbrc.2017.07.007

76. Lee RC, Feinbaum RL, Ambros V. The C. elegans heterochronic gene lin-4 encodes small RNAs with antisense complementarity to lin-14. Cell. (1993) 75:843-54. doi: 10.1016/0092-8674(93)90529-Y

77. Satapathy S, Batra J, Jeet V, Thompson EW, Punyadeera C. MicroRNAs in HPV associated cancers: small players with big consequences. Expert Rev Mol Diagn. (2017) 17:711-22. doi: 10.1080/14737159.2017.1339603
78. Tazawa H, Tsuchiya N, Izumiya M, Nakagama H. Tumor-suppressive miR34a induces senescence-like growth arrest through modulation of the E2F pathway in human colon cancer cells. Proc Natl Acad Sci USA. (2007) 104:15472-7. doi: 10.1073/pnas.0707351104

79. Sun F, Fu H, Liu Q, Tie Y, Zhu J, Xing R, et al. Downregulation of CCND1 and CDK6 by miR-34a induces cell cycle arrest. FEBS Lett. (2008) 582:1564-8. doi: 10.1016/j.febslet.2008.03.057

80. Yamakuchi M, Ferlito M, Lowenstein CJ. miR-34a repression of SIRT1 regulates apoptosis. Proc Natl Acad Sci USA. (2008) 105:13421-6. doi: 10.1073/pnas.0801613105

81. Li B, Hu Y, Ye F, Li Y, Lv W, Xie X. Reduced miR-34a expression in normal cervical tissues and cervical lesions with high-risk human papillomavirus infection. Int J Gynecol Cancer. (2010) 20:597-604. doi: 10.1111/IGC.0b013e3181d63170

82. Hermeking H. MicroRNAs in the p53 network: micromanagement of tumour suppression. Nat Rev Cancer. (2012) 12:613-26. doi: $10.1038 / \mathrm{nrc} 3318$

83. Hünten S, Siemens H, Kaller M, Hermeking H. The p53/microRNA network in cancer: experimental and bioinformatics approaches. Adv Exp Med Biol. (2013) 774:77-101. doi: 10.1007/978-94-007-5590-1_5

84. Wang X, Wang HK, McCoy JP, Banerjee NS, Rader JS, Broker TR, et al. Oncogenic HPV infection interrupts the expression of tumorsuppressive miR-34a through viral oncoprotein E6. RNA. (2009) 15:637-47. doi: 10.1261/rna.1442309

85. Gocze K, Gombos K, Juhasz K, Kovacs K, Kajtar B, Benczik M, et al. Unique microRNA expression profiles in cervical cancer. Anticancer Res. (2013) 33:2561-7.

86. Ribeiro J, Marinho-Dias J, Monteiro P, Loureiro J, Baldaque I, Medeiros R, et al. miR-34a and miR-125b expression in HPV infection and cervical cancer development. Biomed Res Int. (2015) 2015:304584. doi: 10.1155/2015/304584

87. Cortez MA, Ivan C, Valdecanas D, Wang X, Peltier HJ, Ye Y, et al. PDL1 regulation by p53 via miR-34. J Natl Cancer Inst. (2015) 108:djv303. doi: 10.1093/jnci/djv303

88. Wang X, Li J, Dong K, Lin F, Long M, Ouyang Y, et al. Tumor suppressor miR-34a targets PD-L1 and functions as a potential immunotherapeutic target in acute myeloid leukemia. Cell Signal. (2015) 27:443-52. doi: 10.1016/j.cellsig.2014.12.003

89. Mei J, Wang DH, Wang LL, Chen Q, Pan LL, Xia L. MicroRNA$200 \mathrm{c}$ suppressed cervical cancer cell metastasis and growth via targeting MAP4K4. Eur Rev Med Pharmacol Sci. (2018) 22:623-31. doi: 10.26355/eurrev_201802_14286

90. Xi Y, Shalgi R, Fodstad O, Pilpel Y, Ju J. Differentially regulated microRNAs and actively translated messenger RNA transcripts by tumor suppressor p53 in colon cancer. Clin Cancer Res. (2006) 12:2014-24. doi: 10.1158/1078-0432.CCR-05-1853

91. Kao SC, Cheng YY, Williams M, Kirschner MB, Madore J, Lum T, et al. Tumor suppressor microRNAs contribute to the regulation of PD-L1 expression in malignant pleural mesothelioma. J Thorac Oncol. (2017) 12:1421-33. doi: 10.1016/j.jtho.2017.05.024

92. Grenda A, Nicoś M, Szczyrek M, Krawczyk P, Kucharczyk T, Jarosz B, et al. MicroRNAs aid the assessment of programmed death ligand 1 expression in patients with non-small cell lung cancer. Oncol Lett. (2019) 17:5193-200. doi: 10.3892/ol.2019.10207

93. Gibbons DL, Lin W, Creighton CJ, Rizvi ZH, Gregory PA, Goodall GJ, et al. Contextual extracellular cues promote tumor cell EMT and metastasis by regulating miR-200 family expression. Genes Dev. (2009) 23:2140-51. doi: 10.1101/gad.1820209

94. Dyson N, Guida P, Münger K, Harlow E. Homologous sequences in adenovirus E1A and human papillomavirus E7 proteins mediate interaction with the same set of cellular proteins. J Virol. (1992) 66:6893-902. doi: 10.1128/JVI.66.12.6893-6902.1992

95. McLaughlin-Drubin ME, Münger K. The human papillomavirus E7 oncoprotein. Virology. (2009) 384:335-44. doi: 10.1016/j.virol.2008.10.006

96. Karim R, Tummers B, Meyers C, Biryukov JL, Alam S, Backendorf C, et al. Human papillomavirus (HPV) upregulates the cellular deubiquitinase UCHL1 to suppress the keratinocyte's innate immune response. PLoS Pathog. (2013) 9:e1003384. doi: 10.1371/journal.ppat.1003384 
97. Vandermark ER, Deluca KA, Gardner CR, Marker DF, Schreiner $\mathrm{CN}$, Strickland DA, et al. Human papillomavirus type 16 E6 and $\mathrm{E} 7$ proteins alter NF-kB in cultured cervical epithelial cells and inhibition of NF-kB promotes cell growth and immortalization. Virology. (2012) 425:53-60. doi: 10.1016/j.virol.2011. 12.023

98. Xu D, Li J, Li RY, Lan T, Xiao C, Gong P. PD-L1 expression is regulated by NF- $\mathrm{kB}$ during EMT signaling in gastric carcinoma. Onco Targets Ther. (2019) 12:10099-105. doi: 10.2147/OTT.S224053

99. Qin Y, Ekmekcioglu S, Forget M-A, Szekvolgyi L, Hwu P, Grimm EA, et al. Cervical cancer neoantigen landscape and immune activity is associated with human papillomavirus master regulators. Front Immunol. (2017) 8:689. doi: 10.3389/fimmu.2017.00689

100. Hsu PC, Li SH, Yang CT. Recurrent pneumonitis induced by atezolizumab (anti-programmed death ligand 1) in NSCLC patients who previously experienced anti-programmed death 1 immunotherapy-related pneumonitis. J Thorac Oncol. (2018) 13:e227-30. doi: 10.1016/j.jtho.2018.06.022

101. Wakabayashi R, Nakahama Y, Nguyen V, Espinoza JL. The hostmicrobe interplay in human papillomavirus-induced carcinogenesis. Microorganisms. (2019) 7:199. doi: 10.3390/microorganisms 7070199

102. Zhou C, Tuong ZK, Frazer IH. Papillomavirus immune evasion strategies target the infected cell and the local immune system. Front Oncol. (2019) 9:682. doi: 10.3389/fonc.2019. 00682

103. Heeren AM, Rotman J, Stam AGM, Pocorni N, Gassama AA, Samuels $\mathrm{S}$, et al. Efficacy of PD-1 blockade in cervical cancer is related to a CD8+FoxP3+CD25+ T-cell subset with operational effector functions despite high immune checkpoint levels. J Immunother Cancer. (2019) 7:43. doi: 10.1186/s40425-019-0526-z
104. Zhen S, Lu J, Liu Y-H, Chen W, Li X. Synergistic antitumor effect on cervical cancer by rational combination of PD1 blockade and CRISPRCas9-mediated HPV knockout. Cancer Gene Ther. (2019) 27:168-78. doi: 10.1038/s41417-019-0131-9

105. Curie I. Nivolumab in Association With Radiotherapy and Cisplatin in Locally Advanced Cervical Cancers Followed by Adjuvant Nivolumab for up to 6 Months. NiCOL (2017).

106. NCI. Atezolizumab Before and/or With Chemoradiotherapy in Immune System Activation in Patients With Node Positive Stage IB2, II, IIIB, or IVA Cervical Cancer. (2018) Available Online at: https://clinicaltrials.gov/ct2/ show/NCT03738228 (accessed April 02, 2020).

107. Trust RMNF. A Study of Pembrolizumab And Platinum With Radiotherapy in Cervix Cancer (PAPAYA). (2017) Available Online at: https://clinicaltrials. gov/ct2/show/NCT03144466 (accessed 12 Feburary, 2019).

108. Massarelli E, William W, Johnson F, Kies M, Ferrarotto R, Guo M, et al. Combining immune checkpoint blockade and tumor-specific vaccine for patients with incurable human papillomavirus 16-related cancer: a phase 2 clinical trial. JAMA Oncol. (2019) 5:67-73. doi: 10.1001/jamaoncol.201 8.4051

Conflict of Interest: The authors declare that the research was conducted in the absence of any commercial or financial relationships that could be construed as a potential conflict of interest.

Copyright $\odot 2020$ Allouch, Malki, Allouch, Gupta, Vranic and Al Moustafa. This is an open-access article distributed under the terms of the Creative Commons Attribution License (CC BY). The use, distribution or reproduction in other forums is permitted, provided the original author(s) and the copyright owner(s) are credited and that the original publication in this journal is cited, in accordance with accepted academic practice. No use, distribution or reproduction is permitted which does not comply with these terms. 